\title{
NONLINEAR AND COOPERATIVE CONTROL OF MULTIPLE HOVERCRAFT WITH INPUT CONSTRAINTS
}

\author{
William B. Dunbar ${ }^{\dagger}$, Reza Olfati Saber, Richard M. Murray \\ Control and Dynamical Systems, Mail Code 107-81 \\ California Institute of Technology, Pasadena, CA, USA 91125 \\ $\mathrm{Ph}:(626) 395-3366$, Fax: (626) 796-8914 \\ email: $\{$ dunbar, olfati, murray\}@cds.caltech.edu \\ ${ }^{\dagger}$ Corresponding Author
}

\begin{abstract}
Keywords: cooperative control, decentralized control, autonomous multi-vehicles, underactuated systems, unidirectional/bounded control.
\end{abstract}

\begin{abstract}
In this paper, we introduce an approach for distributed nonlinear control of multiple hovercraft-type underactuated vehicles with bounded and unidirectional inputs. First, a bounded nonlinear controller is given for stabilization and tracking of a single vehicle, using a cascade backstepping method. Then, this controller is combined with a distributed gradient-based control for multi-vehicle formation stabilization using formation potential functions previously constructed. The vehicles are used in the Caltech Multi-Vehicle Wireless Testbed (MVWT). We provide simulation and experimental results for stabilization and tracking of a single vehicle, and a simulation of stabilization of a six-vehicle formation, demonstrating that in all cases the control bounds and the control objective are satisfied.
\end{abstract}

\section{Introduction}

Cooperative control of systems with multiple semiautonomous vehicles that are capable of performing coordinated tasks in a distributed manner is an active area of research that has attracted great interest. This is due to the many potential applications, such as coordination of unmanned air vehicles (UAVs), automated search and rescue operations, distributed sensory networks, and automated highway systems to name a few.

An appropriate way to test and demonstrate some of the theoretical results obtained on distributed coordination of multivehicle systems is on a testbed that mimics some of the realistic constraints that exist in real-life applications of interest. To this end, the Caltech Multi-Vehicle Wireless Testbed (MVWT) has been designed, consisting of eight hovercraft-type underactuated vehicles with wireless communication and dedicated sensing, actuation, and computational devices [1]. In this paper, we demonstrate an extension of the result in Olfati Saber and Murray [9] to distributed control of multiple MVWT vehicles, which are subject to bounded and unidirectional controls. This extension relies on an aggressive control design method introduced by the second author in [8] for global configura- tion stabilization of the Vertical Takeoff and Landing (VTOL) aircraft. The dynamics of the VTOL aircraft has certain similarities to the model of a MVWT vehicle.

In Leonard and Fiorelli [6], coordinated control of multiple vehicles with linear and fully-actuated dynamics is examined using potential functions (that differ from the ones used in Olfati Saber and Murray [9]). To the best of our knowledge, distributed cooperative control of multiple hovercraft type vehicles has never been addressed before. In Fantoni et al. [4], control of the kinematic model of a single hovercraft is considered without the constraint of bounded inputs. A rather general result on stabilization of feedforward nonlinear system with bounded controls is given in Teel [12]. This result is not applicable to the underactuated systems considered in this paper because they are in strict feedback form. A direct use of the standard backstepping procedure in Isidori [5] does not lead to construction of uniformly bounded and unidirectional state feedback laws. Instead, we use a variation of backstepping procedure called "cascade backstepping" given in Olfati Saber $[7,8]$ that does not make use of any recursive procedure for construction of Lyapunov functions. An alternative approach that accounts for the control constraints is to use Model Predictive Control (MPC) as in Dunbar et al. [2], where similar restrictive control constraints need to be satisfied for MPC of the Caltech ducted fan experiment. Formation stabilization of three MVWT vehicles using MPC is considered in Dunbar and Murray [3].

In Olfati Saber and Murray [9], a distributed and cooperative control algorithm is developed for formation stabilization of multiple fully-actuated vehicles with double-integrator (linear) dynamics. The key idea of the algorithm in [9] is to automatically obtain formation potentials from rigid and unfoldable formation graphs as defined in Olfati Saber and Murray [10]. Then use a gradient-based controller obtained from these formation potentials to achieve local asymptotic stabilization of a desired formation.

The main contribution of this paper is to show how the method introduced in [9] can be extended to distributed and cooperative control of multiple underactuated vehicles (i.e., hovercraft) with nonlinear dynamics and bounded and unidirectional controls. In fact, global asymptotic stabilization and asymptotic position tracking for the dynamic model of a single underac- 
tuated hovercraft with bounded and unidirectional control is a rather challenging task. Therefore, first we address stabilization/tracking problems for one vehicle. Then, we combine the distributed controller given in [9] with the nonlinear controller designed for a single vehicle to obtain a distributed control algorithm for coordination of multiple underactuated vehicles. This is performed in a way that guarantees the control inputs of each vehicle satisfy the corresponding input constraints throughout formation stabilization and tracking.

The outline of this paper is as follows. In Section 2, stabilization of a single hovercraft is addressed and simulation results are given in Section 3. The control design approach for the tracking controller of a single vehicle is explained in Section 4 with experimental tracking results using the actual MVWT presented in Section 5. The derivation of the distributed algorithm for coordination of multiple vehicles is given in Section 6. Simulation results for six-vehicle formation stabilization is presented in Section 7. Finally, concluding remarks are made in Section 8.

\section{Single Vehicle Stabilization Control}

Each MVWT vehicle rests on omni-directional casters and is powered by two uni-directional ducted fans. The hovercraftlike underactuated dynamics of a MVWT vehicle can be expressed as

$$
\begin{aligned}
\ddot{\boldsymbol{q}} & =\boldsymbol{r}_{1} T-(\eta / m) \dot{\boldsymbol{q}}, \\
\ddot{\theta} & =\tau-(\psi / J) \dot{\theta} \\
F_{1} & =(m T+\tau J / r) / 2 \\
F_{2} & =(m T-\tau J / r) / 2 \\
0 & \leq F_{i} \leq F_{\max }, \quad i=1,2
\end{aligned}
$$

where $(\boldsymbol{q}, \theta) \in \mathbb{R}^{2} \times \mathbb{R}, \boldsymbol{r}_{1}=[\cos (\theta) \sin (\theta)]^{T}$, and $F_{i}$ denotes the unidirectional and bounded force applied by the $i^{\text {th }}$ ducted fan. In addition, $T$ is the thrust and $\tau$ is the torque induced by the forces $F_{1}$ and $F_{2}$. In terms of units, $F_{i}$ are in units of force, while $T$ and $\tau$ are normalized and have units of translational and rotational acceleration, respectively. As a result, $T$ and $\tau$ are restricted to stay in the shaded region specified in Figure 1, where $T_{\max }=2 F_{\max } / m, \tau_{\max }=T_{\max } / 2 \alpha$ and $\alpha=\mathrm{J} / \mathrm{mr}$.

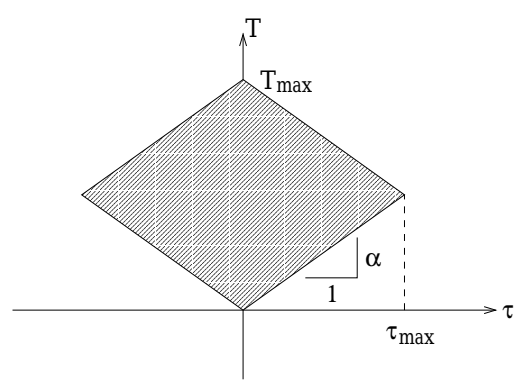

Figure 1: Shaded region depicts allowable thrust force $T$ and torque $\tau$ values.

The parameters $m, J, \eta, \psi, r$ denote the mass, moment of in- ertia, viscous translational and rotational friction coefficients, and the thrust force moment arm, respectively (see [1] for details).

For future use, we define the $m$-dimensional sigmoidal function $\sigma_{m}^{\varepsilon}: \mathbb{R}^{m} \rightarrow \mathbb{R}^{m}$ as

$$
\sigma_{m}^{\varepsilon}(\boldsymbol{y})=\frac{\boldsymbol{y}}{\sqrt{\varepsilon^{2}+\|\boldsymbol{y}\|^{2}}}, \quad \text { and denote } \sigma_{m} \equiv \sigma_{m}^{1},
$$

with $m=1,2, \ldots, \varepsilon>0$ and $\|\cdot\|$ denotes the Euclidean norm $\|\cdot\|_{2}$ unless stated otherwise.

In the following, we construct a controller that stabilizes the translational state in equation (1), i.e. $(\boldsymbol{q}, \dot{\boldsymbol{q}})$, to the origin. The attitude (or rotational) state $(\theta, \dot{\theta})$ is stabilized to $\left(\theta_{C}, 0\right)$, where $\theta_{C}$ is a constant. By our approach, it is not possible to specify $\theta_{C}$ in the stabilization problem. Thus, it should be understood that when we reference stability, we a referring to stabilization to the final state $(\boldsymbol{q}, \theta, \dot{\boldsymbol{q}}, \dot{\theta})=\left(0, \theta_{C}, 0,0\right)$. Remarks on the value of $\theta_{C}$ are given at the end of this section.

Consider translational stabilization to the origin for a doubleintegrator system

$$
\ddot{\boldsymbol{q}}=\boldsymbol{u}-\beta \dot{\boldsymbol{q}}, \quad \beta \geq 0,
$$

subject to the control constraint $\|\boldsymbol{u}\| \leq U_{\max }$. Let $\boldsymbol{u}=$ $\boldsymbol{k}(\boldsymbol{q}, \dot{\boldsymbol{q}})$ be a global stabilizing state-feedback for this 2dimensional double-integrator with damping. To satisfy the control bounds, we use the following saturated (or sigmoidal) PD controller

$$
\boldsymbol{k}(\boldsymbol{q}, \dot{\boldsymbol{q}})=-U_{\max } \sigma_{2}(\boldsymbol{q}+\dot{\boldsymbol{q}}) .
$$

It can be shown that according to LaSalle's Invariance theorem, the smooth and positive-definite function

$$
V(\boldsymbol{q}, \dot{\boldsymbol{q}})=\|\dot{\boldsymbol{q}}\|^{2} / 2+U_{\max } \varphi(\boldsymbol{q}), \quad \varphi(\boldsymbol{q}) \triangleq \sqrt{1+\|\boldsymbol{q}\|^{2}}-1
$$

is a valid Lyapunov function for the closed-loop system that guarantees global asymptotic stability of the equilibrium point $(\boldsymbol{q}, \dot{\boldsymbol{q}})=(0,0)$, where $\varphi$ satisfies $\varphi(\boldsymbol{q}) \geq 0, \varphi(\boldsymbol{q})=0 \Leftrightarrow \boldsymbol{q}=$ 0 and $\nabla \varphi(\boldsymbol{q})=\sigma_{2}(\boldsymbol{q})$.

Given the bounded controller $\boldsymbol{k}$ above and returning to equation (1), the translational dynamics are stabilized by setting

$$
\boldsymbol{r}_{1} T=\boldsymbol{k}, \quad \text { or } \quad T=\|\boldsymbol{k}\|, \boldsymbol{r}_{1}=\boldsymbol{k} /\|\boldsymbol{k}\| \text {. }
$$

From the definition of $\boldsymbol{r}_{1}=[\cos (\theta) \sin (\theta)]^{T}$, the value of $\theta$ and its derivatives can be computed given $\boldsymbol{k}$ and its derivatives. From $\theta$ and its (first two) derivatives, we can in turn determine the torque $\tau$ from the attitude dynamics. As such, derivatives of $\boldsymbol{k} /\|\boldsymbol{k}\|$ are required for construction of the torque $\tau$. In order that these derivatives be well-defined over a domain that includes the origin, we scale $T$ and $\boldsymbol{r}_{1}$ in equation (4) by the parameter $\zeta$ as follows

$$
\hat{\boldsymbol{r}} \equiv \zeta \boldsymbol{r}_{1}, \hat{T} \equiv\left(\frac{T}{\zeta}\right), \quad \text { where } \zeta \equiv \frac{\|\boldsymbol{k}\|}{\sqrt{\|\boldsymbol{k}\|^{2}+\varepsilon^{2}}} \in[0,1)
$$


implying $\hat{\boldsymbol{r}} \hat{T}=\boldsymbol{k}$. We refer to the desired values for $\hat{T}$ and $\hat{\boldsymbol{r}}$ as $\hat{T}_{d}$ and $\hat{\boldsymbol{r}}_{d}$, respectively, given by

$$
\hat{T}_{d}=\sqrt{\|\boldsymbol{k}\|^{2}+\varepsilon^{2}}, \quad \hat{\boldsymbol{r}}_{d}=\sigma_{2}^{\varepsilon}(\boldsymbol{k}) .
$$

We choose the thrust as $T=\zeta \hat{T}_{d}$ so that the control bounds for the thrust are satisfied. In the remainder of this section, the remaining control $\tau$ is constructed, given the desired orientation according to $\boldsymbol{r}_{1}=\hat{\boldsymbol{r}}_{d} / \zeta$ and such that the torque constraints are satisfied. Let $\hat{\boldsymbol{r}}_{d}^{T}=\left[\begin{array}{ll}v_{1} & v_{2}\end{array}\right]$ and $\theta_{d}$ denote the desired value of $\theta$ so that

$$
\zeta \cos \left(\theta_{d}\right)=v_{1}, \quad \zeta \sin \left(\theta_{d}\right)=v_{2} .
$$

Given $\hat{\boldsymbol{r}}_{d}, \theta_{d}=\arctan \left(v_{2} / v_{1}\right)$, which implies the desired values for $\dot{\theta}$ and $\ddot{\theta}$ can be computed as

$$
\begin{gathered}
\dot{\theta}_{d}=\frac{\dot{v}_{2} v_{1}-v_{2} \dot{v}_{1}}{v_{1}^{2}+v_{2}^{2}}=\frac{\dot{v}_{2} v_{1}-v_{2} \dot{v}_{1}}{\zeta^{2}}, \quad \ddot{\theta}_{d}=\frac{\ddot{v}_{2} v_{1}-v_{2} \ddot{v}_{1}}{\zeta^{2}}-\dot{\theta}_{d} \frac{\dot{\zeta}}{\zeta}, \\
\text { where } \quad \dot{\zeta}=\frac{<\boldsymbol{k}, \dot{\boldsymbol{k}}>\left(1-\zeta^{2}\right)}{\zeta\left(\|\boldsymbol{k}\|^{2}+\varepsilon^{2}\right)} .
\end{gathered}
$$

To avoid singularity problems arising from division by $\zeta$, a modified version of $\zeta$ denoted by $\hat{\zeta}$ is used. We define $\hat{\zeta}$ as follows

$$
\hat{\zeta}(\boldsymbol{k}, \varepsilon)=\left\{\begin{array}{ll}
1 / \sqrt{2}, & 0 \leq\|\boldsymbol{k}\|<\varepsilon \\
\zeta, & \varepsilon \leq\|\boldsymbol{k}\|
\end{array} .\right.
$$

Now, we construct the appropriate torque $\tau$. Writing the attitude error state as $\left(\theta_{e}, \dot{\theta}_{e}\right)=\left(\theta-\theta_{d}, \dot{\theta}-\dot{\theta}_{d}\right)$ and combining this with equation (1) yields

$$
\begin{gathered}
\ddot{\theta}_{e}+\ddot{\theta}_{d}=\tau-(\psi / J)\left(\dot{\theta}_{e}+\dot{\theta}_{d}\right) \Longleftrightarrow \ddot{\theta}_{e}=\hat{\tau}-(\psi / J) \dot{\theta}_{e}, \\
\text { where } \hat{\tau} \equiv \tau-\ddot{\theta}_{d}-(\psi / J) \dot{\theta}_{d} .
\end{gathered}
$$

We use the following saturated PD controller to stabilize the above error dynamics

$$
\hat{\tau}=-B \sigma_{1}\left(\theta_{e}+\dot{\theta}_{e}\right),
$$

that satisfies the torque constraint $|\hat{\tau}| \leq B$. Finally, the desired torque for the original system is

$$
\tau_{d}=-B \sigma_{1}\left(\theta_{e}+\dot{\theta}_{e}\right)+\ddot{\theta}_{d}+(\psi / J) \dot{\theta}_{d} .
$$

According to equation (3) and $T=\hat{\zeta} \hat{T}_{d}$, the bounds on the desired thrust are $\varepsilon / \sqrt{2} \leq T \leq U_{\max }$, so choosing $\varepsilon$ small enough and $U_{\max }=T_{\max }$ will always generate a $T$ that satisfies the aforementioned bounds. The torque bounds are captured by the following function that is parameterized by the thrust $T$

$$
|\tau| \leq a(T), \quad \text { where } a(T)=\tau_{\max }-\frac{1}{\alpha}\left|T-T_{\max } / 2\right| .
$$

The torque bounds could be achieved in practice by incorporating a saturation function, namely by applying the torque $\tau=\sigma_{1}\left(\tau_{d}\right) a(T)$, which we refer to as torque cutting.
For tuning the performance of the controller, we introduce parameters in $\boldsymbol{k}$ and $\hat{\tau}$ as

$$
\boldsymbol{k}=-T_{\max } \sigma_{2}\left(\alpha_{1} \boldsymbol{q}+\alpha_{2} \dot{\boldsymbol{q}}\right), \quad \hat{\tau}=-B \sigma_{1}\left(\beta_{1} \theta_{e}+\beta_{2} \dot{\theta}_{e}\right) .
$$

The five controller parameters are thus $\left(\alpha_{1}, \alpha_{2}, \beta_{1}, \beta_{2}, B\right)$. These parameters can be chosen such that $\left|\tau_{d}\right| \leq a(T)$, in which case we can set $\tau=\tau_{d}$ and the constraints in equation (1) are satisfied analytically. In this case, a proof of asymptotic stability of the closed-loop dynamics follows along the same lines of the proof of Theorem 2 in [8].

In the stabilization simulation, the parameters are chosen such that the torque bounds are satisfied analytically (meaning without cutting but for a given bounded set of initial conditions) and we implement $\tau=\tau_{d}$. Of course, for large enough initial state error, the same controller parameters result in violation of the torque bounds. However, it should be noted that the MVWT platform has a bounded configuration space and the simulation result here shows aggressive performance with a non-trivial initial state error. For tracking, stability holds when an appropriate time-parameterization of the desired trajectory, with uniformly bounded tracking path curvature, is chosen. Although torque cutting will guarantee that the torque bounds are respected, independent of the controller parameters, such a control law is known to be non-robust and not necessarily stabilizing. This is why it is desirable to avoid torque cutting.

As stated, stabilization is with respect to the final state $(\boldsymbol{q}, \theta, \dot{\boldsymbol{q}}, \dot{\theta})=\left(0, \theta_{C}, 0,0\right)$. With the notation $\boldsymbol{q}=(x, y)$, $\theta_{d}=\arctan \left(v_{2} / v_{1}\right)=\arctan \left(\left(\alpha_{1} y+\alpha_{2} \dot{y}\right) /\left(\alpha_{1} x+\alpha_{2} \dot{x}\right)\right)$, and so $\left(\theta_{d}, \dot{\theta}_{d}\right)$ exhibits initial transient behavior and converges to $\left(\theta_{C}, 0\right)$, where $\theta_{C}=\lim _{t \rightarrow \infty} \arctan \left(\left(\alpha_{1} y+\alpha_{2} \dot{y}\right) /\left(\alpha_{1} x+\right.\right.$ $\left.\left.\alpha_{2} \dot{x}\right)\right)$. The value of this limit depends upon the initial translational state and the controller parameters $\left(\alpha_{1}, \alpha_{2}\right)$, and therefore cannot be commanded in general to take any desired value.

\section{Single Vehicle Stabilization Simulation}

The physical parameter values [1] are $(m, J, \eta, \psi, r)=(5.15$ $\mathrm{kg}, 0.05 \mathrm{~kg}-\mathrm{m}^{2}, 4.5 \mathrm{~kg} / \mathrm{s}, 0.084 \mathrm{~kg} \mathrm{~m}^{2} / \mathrm{s}, 0.124 \mathrm{~m}$ ). Figure 2 shows the response of the vehicle from initial condition $(\boldsymbol{q}, \theta, \dot{\boldsymbol{q}}, \dot{\theta})=(1,1, \pi / 4,0,0,0)$ to the origin. The graphical picture of the vehicle shows the position, orientation and the fan forces, where the length of the cone is proportional to the force in each fan. Figure 3 shows the inputs on the force/torque map and Figure 4 shows the closed-loop state responses. In this case, $\tau=\tau_{d}$ and the torque bounds are analytically satisfied. The dashed lines in the state responses define the desired state $\left(0, \theta_{d}, 0, \dot{\theta}_{d}\right)$. The controller parameter values chosen for this simulation are $\left(\alpha_{1}, \alpha_{2}, \beta_{1}, \beta_{2}, B\right)=$ $\left(0.5,0.75,0.2,0.05, \tau_{\max } / 2\right)$. The closed-loop response attests to the stability and performance of the controller. The initial torque (visible in the difference in the length of the fan cones and on the force map) results from the initial configuration error, particularly the angle error $\theta_{e}(0) \neq 0$. 


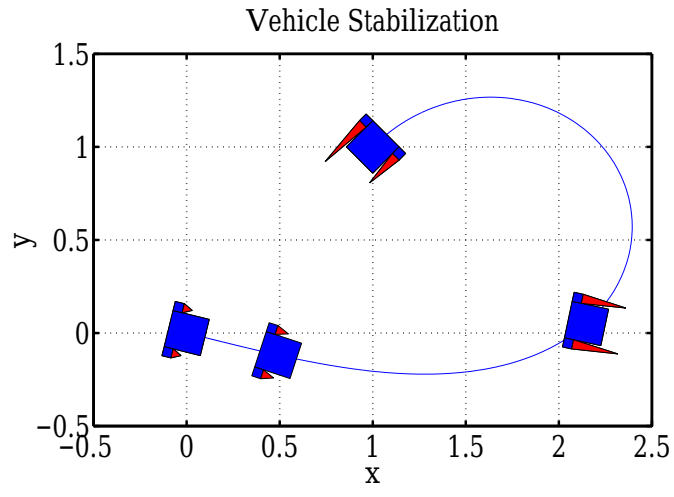

Figure 2: Stabilization of vehicle in $\boldsymbol{q}=(x, y)$ space from $(1,1, \pi / 4,0,0,0)$ to the origin.

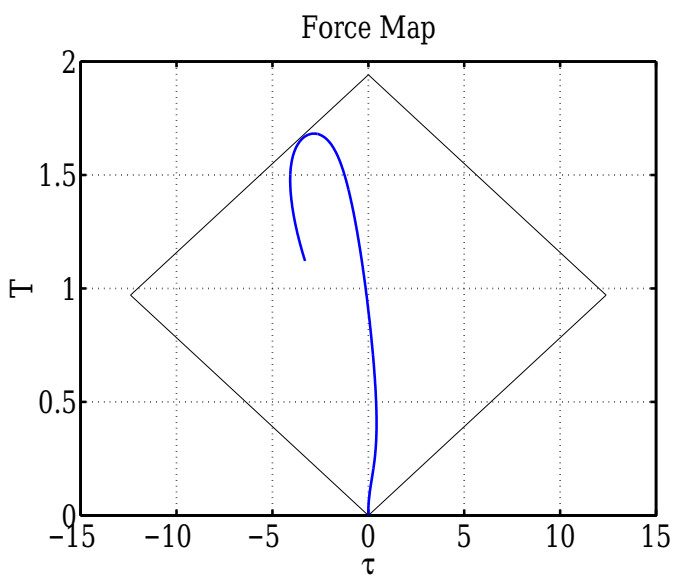

Figure 3: The force/torque map.
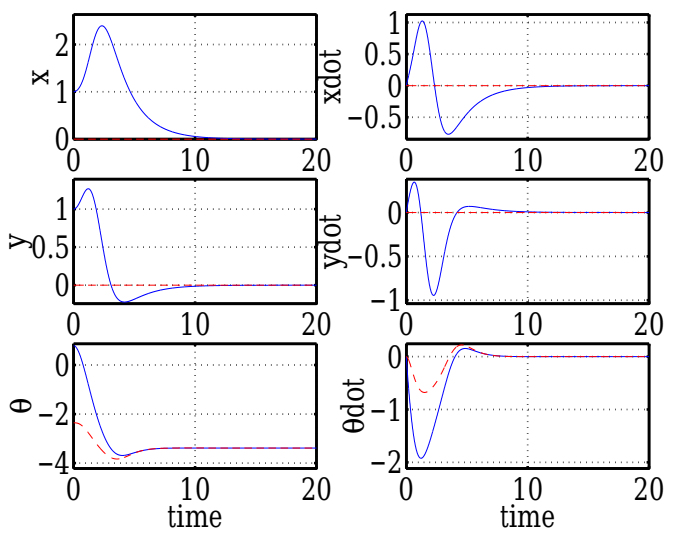

Figure 4: Closed-loop response of the vehicle states (solid lines) and desired state $\left(0, \theta_{d}, 0, \dot{\theta}_{d}\right)$ (dashed lines).

\section{Single Vehicle Tracking Control}

Consider tracking a desired translational position and velocity $\left(\boldsymbol{q}_{d}, \dot{\boldsymbol{q}}_{d}\right)$ with double integrator dynamics $\ddot{\boldsymbol{q}}=\boldsymbol{u}-\beta \dot{\boldsymbol{q}}$, for some $\beta \geq 0$, subject to $\|\boldsymbol{u}\| \leq U_{\max }$. Again, denote the controller $\boldsymbol{u}=\boldsymbol{k}\left(\boldsymbol{q}, \dot{\boldsymbol{q}}, \boldsymbol{q}_{d}, \dot{\boldsymbol{q}}_{d}\right)$ and the translational error state $\left(\boldsymbol{q}_{e}, \dot{\boldsymbol{q}}_{e}\right)=$ $\left(\boldsymbol{q}-\boldsymbol{q}_{d}, \dot{\boldsymbol{q}}-\dot{\boldsymbol{q}}_{d}\right)$. Using a saturated PD error controller, the control law is given by

$$
\boldsymbol{k}=-\hat{U}_{\max } \sigma_{2}\left(\alpha_{1} \boldsymbol{q}_{e}+\alpha_{2} \dot{\boldsymbol{q}}_{e}\right)+\ddot{\boldsymbol{q}}_{d}
$$

To satisfy the thrust constraint, we must characterize the parameter $\hat{U}_{\max }$ and the desired translational acceleration. For example, when $\left\|\ddot{\boldsymbol{q}}_{d}\right\| \leq \mu T_{\text {max }}$, for some $\mu \in(0,1 / 2]$, the appropriate choice is $\hat{U}_{\max }=T_{\max }(1-\mu)$. The control law for $T$ is as defined in the previous section with $\boldsymbol{k}$ given above. The expression for $\tau$ is also the same as in the previous section, although to guarantee that the configuration error can be driven to zero in the presence of equation (5), the values for $\boldsymbol{q}_{d}^{(3)}$ and $\boldsymbol{q}_{d}^{(4)}$ (denoting third and fourth time derivatives), as well as the controller parameters $\left(\alpha_{1}, \alpha_{2}, \beta_{1}, \beta_{2}, B\right)$, must also be characterized. The specific characterizations will be detailed in a future paper that addresses stability theoretically [11].

\section{Single Vehicle Tracking Experiment}

The tracking signal is $\boldsymbol{q}_{d}=2.5(\sin (\omega t), \cos (3 \omega t))$, where $\omega=0.16 \mathrm{rad} / \mathrm{sec}$ is chosen such that $\left\|\ddot{\boldsymbol{q}}_{d}\right\| \leq 0.3 T_{\text {max }}$. Figure 5 shows a plot of the hovercraft trajectory (solid line) and the reference trajectory (dashed line) in $x, y$ space. The circle and diamond indicate the initial and final positions of the hovercraft, respectively. The plot also shows the dimensions of the

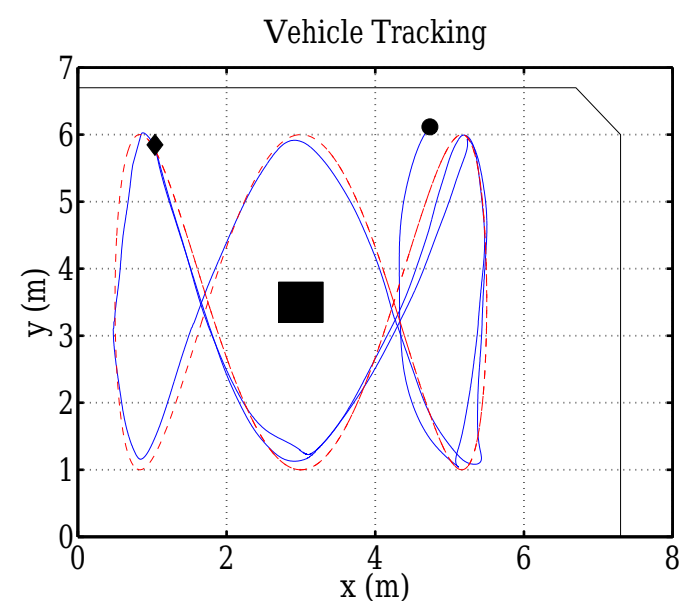

Figure 5: Tracking reference in $(x, y)$ space of hovercraft on MVWT floor.

MVWT floor [1] on which the vehicles are permitted to move; the outer line denotes the floor boundary and the inner box denotes an obstacle that the hovercraft is not permitted to pass through. Figure 6 shows the state responses and reference trajectories. The closed-loop response attests to the performance of the controller in the presence of the uncertainty and disturbances present in the MVWT experiment. 

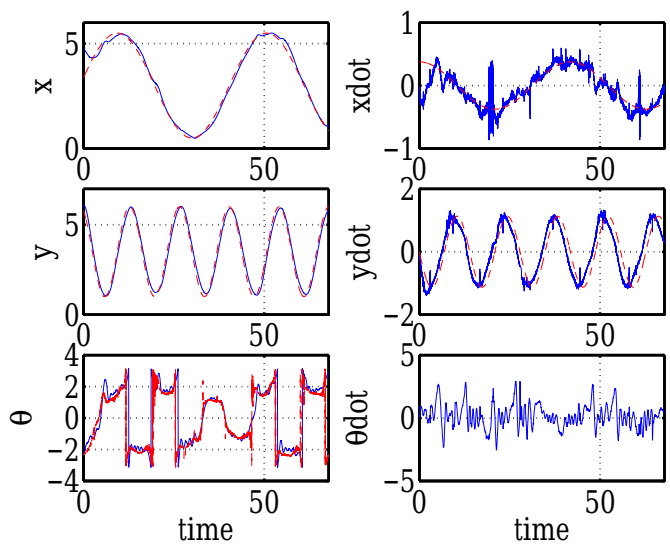

Figure 6: Closed-loop response of the experimental vehicle states.

\section{Multi-vehicle Formation Potential Based Co- operative Control}

From [9], a cooperative and distributed control law for the translational dynamics of the $i$ th vehicle in a rigid formation graph is given in explicit form $\boldsymbol{k}_{i}=\boldsymbol{k}_{i}\left(\boldsymbol{q}_{i}, \dot{\boldsymbol{q}}_{i}, \boldsymbol{q}_{\mathcal{N}_{i}}\right)$ by

$$
\boldsymbol{k}_{i}=T_{\text {max }}\left[\lambda /\left|\mathcal{N}_{i}\right|\left(\sum_{j \in \mathcal{N}_{i}} \sigma_{1}\left(\eta_{i j}\right) \boldsymbol{n}_{i j}\right)-(1-\lambda) \sigma_{2}\left(\dot{\boldsymbol{q}}_{i}\right)\right] \text {, }
$$

where $\lambda \in(0,1), \boldsymbol{q}_{\mathcal{N}_{i}}$ are translational configuration variables, $\mathcal{N}_{i}$ denotes the set of neighbors of the $i$ th vehicle on the graph, $\left|\mathcal{N}_{i}\right|$ is the total number of neighbors of the $i$ th vehicle, and the edge shape variables and the unit vectors connecting vehicle $i$ to vehicle $j$ are, respectively, defined by

$$
\eta_{i j}=\left\|\boldsymbol{q}_{j}-\boldsymbol{q}_{i}\right\|-d_{i j}, \quad \boldsymbol{n}_{i j}=\frac{\boldsymbol{q}_{j}-\boldsymbol{q}_{i}}{\left\|\boldsymbol{q}_{j}-\boldsymbol{q}_{i}\right\|}
$$

The feedback laws $T_{i}, \tau_{i}$ for each vehicle $i$ are again as defined in Section 2 with $\boldsymbol{k}$ set to $\boldsymbol{k}_{i}$. Proof that $\boldsymbol{k}_{i}$ is stabilizing and that $\left\|\boldsymbol{k}_{i}\right\| \leq T_{\text {max }}$, for all vehicles $i$, is detailed in [9]. The controller parameters are $\left(\alpha_{1}, \alpha_{2}, \lambda, \beta_{1}, \beta_{2}, B\right)$, which can be chosen independently for each vehicle in the formation.

As before, the derivation of the torque $\tau_{i}$ requires two timederivatives of $\boldsymbol{k}_{i}$, which in turn requires that each vehicle have the following information from its neighbors at each update of the controller: $\left(\boldsymbol{q}_{j}, \theta_{j}, \dot{\boldsymbol{q}}_{j}, \boldsymbol{k}_{j}\right)$, for all $j \in \mathcal{N}_{i}$. Thus, the control law is generated in two-stages (in series). That is, for vehicle $i, \boldsymbol{k}_{i}$ is first computed given state $i$ and the position of all neighbors, then the rest of the control law is computed given $\left(\boldsymbol{q}_{j}, \theta_{j}, \dot{\boldsymbol{q}}_{j}, \boldsymbol{k}_{j}\right)$, for all $j \in \mathcal{N}_{i}$. In a practical setting, this means (wireless) communication must occur amidst the control computations.

\section{Six-Vehicle Formation Stabilization Simula- tion}

The directed graph $G=(V, E)$ that corresponds to this example has vertex and edge sets $V=\{1,2,3,4,5,6\}$ and $E=$ $\{(1,2),(1,3),(2,3),(2,5),(3,2),(3,5),(4,2),(4,5),(5,2)$, $(5,3),(6,5),(6,3)\}$. Figure 7 shows the desired formation depicted as a directed graph, where each vehicle has 2 neighbors with a desired distance of 1 from each neighbor. Figure 8 shows the response of the six vehicles for stabilization from the initial configuration shown, with zero initial velocity.

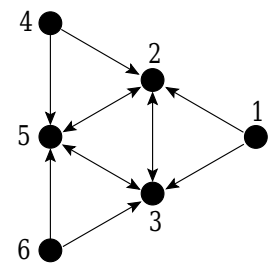

Figure 7: Desired formation, depicted as a directed graph.

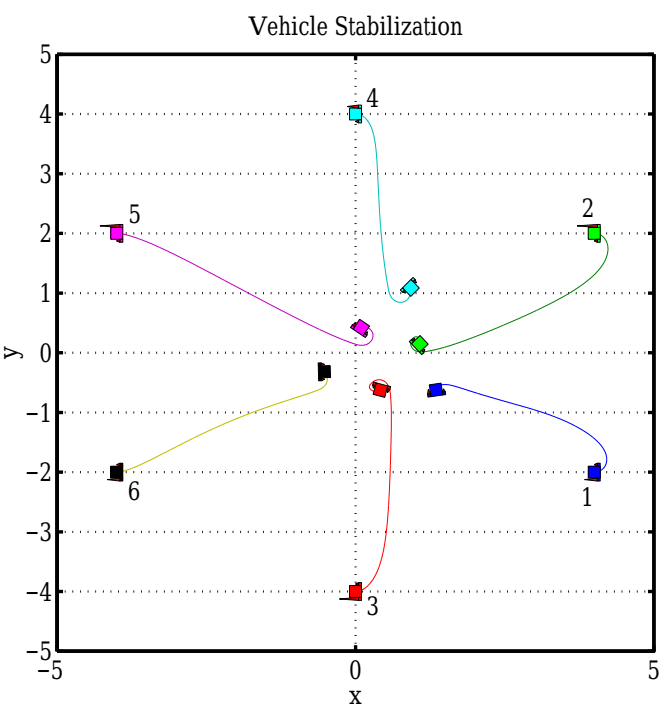

Figure 8: Six vehicle stabilization response.

Figure 9 shows the six input responses on the force/torque map and Figure 10 shows the inter-vehicle distances for each edge in the graph. The controller parameter values chosen for this simulation, common for all six vehicles, are $\left(\alpha_{1}, \alpha_{2}, \lambda, \beta_{1}, \beta_{2}, B\right)=\left(1.0,0.5,0.5,1.0,0.5, \tau_{\max } / 2\right)$.

\section{Conclusions}

Cooperative and distributed nonlinear control of a multivehicle formation that consists of the underactuated hovercrafttype vehicles of the Caltech MVWT has been examined in this paper. The nonlinear hovercraft controllers that perform stabilization/tracking for a single underactuated vehicle were here 


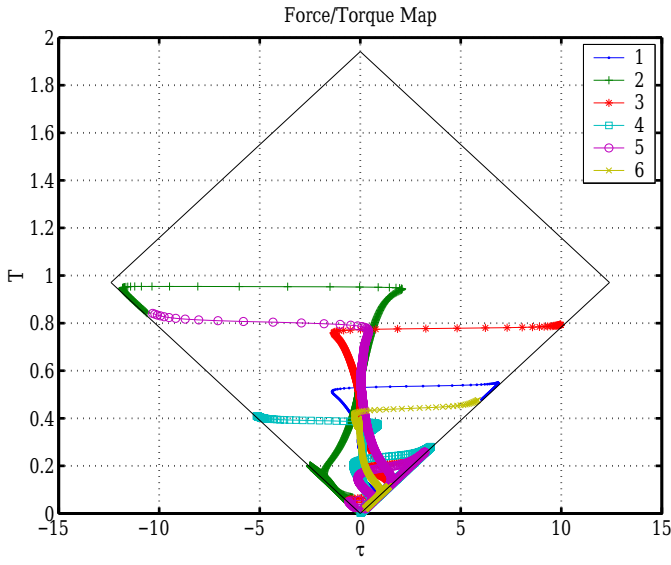

Figure 9: The force/torque map.

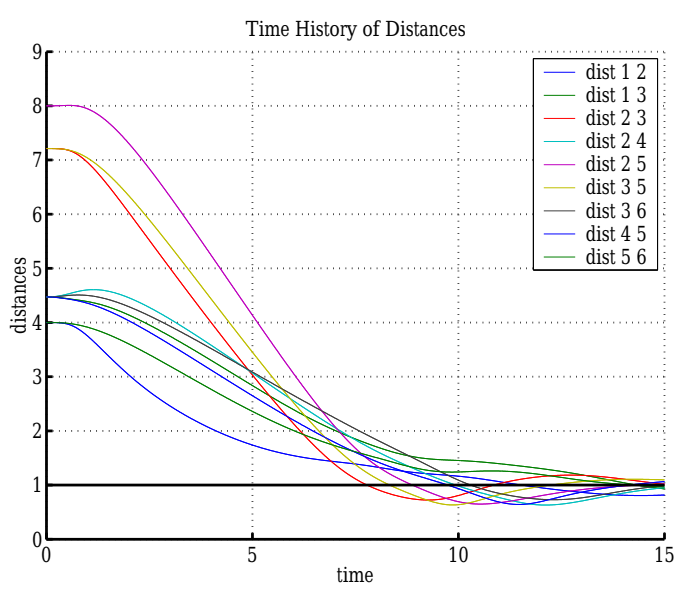

Figure 10: Intervehicle distances for all edges in graph.

developed according to the cascade backstepping method given in [8]. Then, the distributed control algorithm introduced in [9] for asymptotic formation stabilization of multiple vehicles with double-integrator type dynamics, was combined with the nonlinear hovercraft controller. The result is a distributed nonlinear control algorithm for formation stabilization of multiple underactuated, nonlinear hovercraft-type vehicles subject to bounded and unidirectional input constraints.

We presented simulation and experimental results for stabilization and trajectory tracking, respectively, of a single vehicle, and a stabilization simulation of a six vehicle formation. In all cases, we observed that the controllers perform well, in particular behaving in a rather aggressive way by initially staying close to the control bounds. Moreover, all controllers satisfy the control bounds imposed on the inputs of each vehicle.

Formal proofs of stability analysis is the topic of an upcoming paper [11] and follows the same lines of argument presented in $[8,9]$. Additional experimental results, for single and multiple vehicles, will also be conducted in the near future.

\section{Acknowledgements}

Partial support for this work was provided by DARPA under grant F33615-98-C-3613. The authors thank Steve Waydo for his help with the experimental results.

\section{References}

[1] L. Cremean, W.B. Dunbar, D. van Gogh, J. Meltzer, R.M. Murray, E. Klavins, and J. Hickey. The Caltech multi-vehicle wireless testbed. In Proceedings of the Conference on Decision and Control, Las Vegas, NV, 2002.

[2] W. B. Dunbar, M. B. Milam, R. Franz, and R. M. Murray. Model predictive control of a thrust-vectored flight control experiment. In Proceedings of the IFAC World Congress, Barcelona, Spain, 2002.

[3] W. B. Dunbar and R. M. Murray. Model predictive control of coordinated multi-vehicle formations. In Proceedings of the Conference on Decision and Control, Las Vegas, NV, 2002.

[4] I. Fantoni, R. Lozano, F. Mazenc, and K.Y. Pettersen. Stabilization of a nonlinear underactuated hovercraft. Int. J. Robust Nonlinear Control, 10:645-654, 2000.

[5] A. Isidori. Nonlinear Control Systems. Springer, 1995.

[6] N. E. Leonard and E. Fiorelli. Virtual leaders, artificial potentials and coordinated control of groups. In 2001 Conference on Decision and Control, Florida, 2001.

[7] R. Olfati-Saber. Nonlinear Control of Underactuated Mechanical Systems with Applications to Robotics and Aerospace Vehicles. PhD thesis, Massachusetts Institute of Technology, Department of Electrical Engineering and Computer Science, February 2001, http: //www. cds . caltech. edu/ ol fati.

[8] R. Olfati-Saber. Global configuration stabilization for the VTOL aircraft with strong input coupling. IEEE Trans. Auto. Contr., 47(11):1949-1952, November, 2002.

[9] R. Olfati-Saber and R. M. Murray. Distributed cooperative control of multiple vehicle formations using structural potential functions. In Proceedings of the IFAC World Congress, Barcelona, Spain, 2002.

[10] R. Olfati-Saber and R. M. Murray. Graph Rigidity and Distributed Formation Stabilization of Multi-Vehicle Systems. In Proceedings of the IEEE Int. Conference on Decision and Control, Las Vegas, Nevada, Dec. 2002, http://www.cds. caltech.edu/ olfati/papers.

[11] R. Olfati Saber. Stability analysis for formations of multiple hovercraft with bounded inputs. In Preparation.

[12] A. R. Teel. Using saturation to stabilize single-input partially linear composite nonlinear systems. In Proc. IFAC Nonlinear Contr. Syst. Design Symp., pages 224-229, 1992. 